\title{
Une formation inter professionnelle pour apprendre à travailler ensemble. La perception des étudiants en santé
}

\author{
An interprofessional training program to learn to work together. \\ The perception of health care students.
}

\author{
Laure FIQUET ${ }^{1}$, Sandrine HUGE ${ }^{1}$, Françoise ANNEZO ${ }^{2}$, Anthony CHAPRON ${ }^{1}$, \\ Emmanuel ALLORY ${ }^{1}$, Pierric RENAUT ${ }^{1}$ \\ 1 Département de médecine générale, Université de Rennes 1, France \\ 2 Association française pour le développement de l'éducation thérapeutique (AFDET)
}

Manuscrit reçu le 22 décembre 2014 ; commentaires éditoriaux formulés aux auteurs le 9 mai 2015 ; accepté pour publication le 20 juillet 2015

\begin{abstract}
Mots-clés
éducation interprofessionnelle ; formation initiale ; collaboration ; interprofessionnalité

Résumé - Contexte : La prise en charge des maladies chroniques impose aux professionnels de travailler ensemble, dans un souci de qualité des soins. L'éducation interprofessionnelle vise à préparer à ces pratiques collaboratives. Une formation interprofessionnelle a été mise en place auprès de 110 étudiants de 8 professions de santé à Rennes. Objectif : Notre étude vise à analyser la perception des étudiants concernant cette formation et l'impact éventuel dans leur pratique professionnelle. Méthode : Une méthode qualitative par entretiens semi-directifs a été conduite par deux chercheurs, auprès d'étudiants deux ans après leur participation à la formation. Une analyse thématique manuelle avec double codage a été réalisée en identifiant les unités de sens puis en catégorisant les thèmes retrouvés. Résultats : Seize entretiens semidirectifs ont été réalisés auprès d'étudiants issus de sept filières de formation. Cette formation est novatrice. Elle est une occasion unique de rencontrer les autres professionnels de santé. Elle permet de s'interroger sur les notions d'identité professionnelle et de représentations. La découverte des compétences de chacun mais aussi le développement de relations plus égalitaires entre les différents acteurs de santé sont des éléments essentiels. Si l'impact d'une telle formation est difficile à évaluer, les discours des étudiants mettent en exergue une nette volonté de développer le travail collaboratif. Les étudiants veulent s'engager et construire des modes d'exercice collaboratifs dans leur milieu professionnel ambulatoire ou hospitalier. Un changement de regard sur la pratique est souligné. Conclusion : Les formations interprofessionnelles apparaissent indispensables pour rencontrer les autres professionnels de santé, déconstruire les représentations et travailler dans une logique de collaboration interprofessionnelle.
\end{abstract}




$\begin{array}{ll}\begin{array}{l}\text { Keywords } \\ \text { interprofessional }\end{array} & \text { Abstract - Background: Health care professionals are expected to collaborate and provide } \\ \text { education; } & \text { quality care in the management of chronic diseases. Interprofessional education is designed to } \\ \text { medical education; } & \text { train professionals as collaborative-ready practitioners. In Rennes an interprofessional pro- } \\ \text { collaboration; } & \text { gram was introduced and included } 110 \text { students from } 8 \text { health professions. Aim: Our qualita- } \\ \text { interprofessionalism } & \text { tive study proposes to explore students' perceptions of the training program and the impact on } \\ & \text { their practice. Methods: Two researchers conducted semi-structured interviews with students } \\ & \text { two years after their involvement in the program. The researchers independently coded the } \\ \text { data manually and analysed the transcripts according to themes. Results: Sixteen interviews } & \text { with students from } 7 \text { health care professions were conducted. This course is innovative in that } \\ & \text { it provides students with their only chance to meet other health care students while offering } \\ & \text { an opportunity to challenge professional identity and representations. Even though it is diffi- } \\ & \text { cult to evaluate the full impact of the training, recognizing individual skills and establishing } \\ \text { more equal relationships appear to be key drivers in collaborative practices. Students want to } & \text { be involved and build collaborative practices. Their views about their skills are evolving. } \\ \text { Conclusion: Interprofessional education programs are needed to learn about other health care } & \text { professionals and their skills, clear up misconceptions and enhance collaborative attitudes. }\end{array}$

\section{Introduction}

Collaboration professionnelle dans le champ de la santé et formation interprofessionnelle

La prise en charge des maladies chroniques impose aux professionnels de travailler ensemble, dans un souci de qualité des soins ${ }^{[1,2]}$. D'Amour définit la collaboration professionnelle en santé comme «les relations et les interactions entre professionnels leur permettant de partager leurs connaissances, leur expertise et leur expérience pour les mettre au service des patients ${ }^{[3]}$. La collaboration est indispensable dans l'organisation des soins. Cela nécessite notamment de définir le rôle de chacun et de reconnaître ses compétences $^{[4]}$.

Les universités et autres instituts de formation en santé doivent s'adapter aux rôles nouveaux des professionnels de santé. Le Consensus mondial sur la responsabilité sociale des facultés de médecine préconise ainsi la création de partenariats avec la société civile. Il s'agit de former des professionnels capables de partager efficacement des compétences et de déléguer des tâches ${ }^{[5]}$. L'acquisition de compétences dans ces domaines est prévue dans les programmes de formation. Par exemple, les étudiants de médecine générale doivent acquérir des compétences pour coordonner la prise en charge du patient avec les autres acteurs médico-sociaux ${ }^{[6]}$. Les étudiants en soins infirmiers doivent être capables d'organiser et coordonner des interventions soignantes. Pourtant, les cursus de formation des professionnels de santé sont pour la plupart cloisonnés et uni-professionnels. Une des perspectives d'amélioration consiste à promouvoir des formations interprofessionnelles, définies lorsqu'au moins deux professions s'engagent dans des processus d'apprentissage conjoints, réciproques, qui leur permettent de mieux se connaître et de collaborer dans une visée d'amélioration de la qualité des soins $^{[7,8]}$. Ces formations sont nécessaires pour préparer des pratiques collaboratives qui répondent aux besoins de santé ${ }^{[9]}$.

\section{De la discipline à l'inter professionnalité}

Les concepts de pluridisciplinarité et de pluriprofessionnalité sont souvent utilisés sans réelle distinction. Le terme de discipline renvoie principalement à un construit social, visant à créer un espace de significations partagées possédant ses propres règles. Morin définit la discipline comme " une catégorie organisationnelle au sein de la connaissance scientifique; elle y institue la division et la spécialisation du travail.» ${ }^{[10]}$

Dans le champ de la santé, certains professionnels ne se reconnaissent pas dans la notion de discipline, ils ne se reconnaissent pas dans les disciplines médicales. Les soignants, quelles que soient leurs 
activités sont des professionnels, au sens où ils exercent un métier qui nécessite une qualification professionnelle et une spécialisation ${ }^{[11]}$. Dans le champ de la santé, la pluri professionnalité témoigne ainsi d'une activité où plusieurs professionnels participent et travaillent conjointement. L'inter professionnalité, elle, implique une réelle interaction entre les différents professionnels où chacun a sa place. Il s'agit alors de passer d'une logique de professionnalisation à une logique de collaboration ${ }^{[3]}$. La formation interprofessionnelle implique ainsi de se départir de sa fonction professionnelle et d'envisager les perspectives des autres professions. Une formation trans professionnelle consiste à mener une formation interprofessionnelle dans un contexte réel de pratique clinique $^{[8]}$, ce qui permet aux étudiants d'ancrer leurs apprentissages dans la réalité, avec des situations authentiques complexes.

Une expérimentation de formation interprofessionnelle

Constatant les difficultés de collaboration entre professionnels de santé en pratique, la coordinatrice d'un réseau de santé (F. Annezo) a imaginé une formation interprofessionnelle à Rennes. Onze instituts de formation ont été sollicités. Un formateur par institut a été impliqué pour construire le programme et animer la session de formation. Un formateur extérieur a apporté son expertise concernant la pédagogie.

Il s'agissait de mettre en place une formation au travail interprofessionnel centré sur le patient. L'intention pédagogique était d'enseigner le «travailler ensemble ».

Les compétences visées par cette formation étaient que les étudiants se forment à une pratique collaborative et au travail interprofessionnel. Ainsi, il fallait envisager les différents aspects d'un travail en inter professionnalité : difficultés, avantages, aspects pratiques pour comprendre le rôle de chacun dans un travail collaboratif. Il s'agissait également pour les apprenants de connaître les différentes professions de santé, leurs compétences propres et partagées, pour en faire des partenaires de travail.

La formation concernait 110 étudiants futurs professionnels de santé (diététiciens, ergothérapeutes, infirmiers, masseurs-kinésithérapeutes, médecins généralistes, pharmaciens, podologues, sages-femmes), qui étaient le plus souvent volontaires. Les formateurs avaient choisi de réunir des étudiants terminant leur cursus un an après le début de la formation. Ainsi, en fonction de leur profession, ils étaient en deuxième année d'études d'infirmier ou de masseur-kinésithérapeute, en troisième ou quatrième semestre d'internat de médecine générale, en cinquième année de pharmacie filière officine, etc. Ce choix a été réalisé dans l'optique de former des professionnels qui seraient diplômés en même temps, quelle que soit leur formation initiale et qui pourraient donc être amenés à travailler ensemble dès l'obtention de leur diplôme.

La première formation a eu lieu à Rennes, entre octobre 2009 et octobre 2010, à raison de trois séminaires de deux jours, dédiés chacun à un thème autonome. Le premier thème abordait les différentes professions de santé et les collaborations développées. Le second concernait la maladie chronique et la relation soignant soigné. Le troisième s'intéressait à l'éducation thérapeutique du patient comme exemple de pratique interprofessionnelle. Cette formation a ensuite été poursuivie à Rennes, depuis 2009, une fois par an, réunissant toujours plus d'une centaine d'étudiants. Elle a été également développée par l'Association française pour le développement de l'éducation thérapeutique (AFDET) dans d'autres villes et le contenu pédagogique a été adapté à partir des expériences précédentes.

\section{La construction pédagogique}

Cette formation a été construite en inter professionnalité par les formateurs des différents instituts. La première étape était de déterminer les besoins des apprenants. Centrer les enseignements sur les apprenants a guidé la construction de la formation, en se basant sur le paradigme de l'apprentissage ${ }^{[12]}$. Faire émerger les connaissances antérieures des apprenants et leurs représentations ${ }^{[13]}$ en début de formation était souhaité. Ensuite, des activités pédagogiques permettant de construire, d'élaborer du sens pour en faire des apprentissages signifiants, étaient mises en œuvre. Ces activités pédagogiques, développées à partir de tâches professionnelles, étaient contextualisées et 
Tableau I. Exemple de séquences pédagogiques de la 1ere session de formation.

\begin{tabular}{|l|l|l|}
\hline $\mathrm{N}^{\circ}$ & Objectif pédagogique & Techniques pédagogiques \\
\hline 1 & $\begin{array}{l}\text { Prendre conscience de la } \\
\text { conception que l'on a des autres } \\
\text { métiers. }\end{array}$ & $\begin{array}{l}\text { Technique pédagogique : Travail de groupe organisé par } \\
\text { profession. « Etablir à l'issue une carte d'identité de chacun des autres corps } \\
\text { de métier » }\end{array}$ \\
\hline 2 & $\begin{array}{l}\text { Etre capable de décrire ce qu'est } \\
\text { une représentation et ses } \\
\text { conséquences sur le travail en } \\
\text { interdisciplinarité. }\end{array}$ & $\begin{array}{l}\text { Intervention d'un sociologue de la santé sur les représentations et la notion } \\
\text { d'identité professionnelle. }\end{array}$ \\
\hline 3 & $\begin{array}{l}\text { Etre capable de décrire le travail } \\
\text { de son corps de métier, les liens } \\
\text { avec les autres et ce qu'ils peuvent } \\
\text { s'apporter mutuellement. }\end{array}$ & $\begin{array}{l}\text { « Speed dating » : 8 Entrevues de 7 minutes entre individus de profession } \\
\text { différentes, avec pour « mission » de décrire son travail, son quotidien, et de } \\
\text { convaincre l'autre de travailler en lien avec lui au service du patient. }\end{array}$ \\
\hline 4 & $\begin{array}{l}\text { Etre capable de décrire le travail } \\
\text { de chacun des corps de métier } \\
\text { représenté, et les liens avec son } \\
\text { propre métier. }\end{array}$ & $\begin{array}{l}\text { Travail de groupe : faire la synthèse de l'exercice précédent, ce qu'ils ont } \\
\text { appris, ce qui les a surpris, les nouveautés, comment ils peuvent travailler } \\
\text { ensemble. }\end{array}$ \\
\hline 5 & $\begin{array}{l}\text { Prendre conscience des } \\
\text { représentations de sa profession } \\
\text { par les autres. }\end{array}$ & $\begin{array}{l}\text { Théâtre : Saynètes type « commedia dell' arte » Sur 3 situations de prise en } \\
\text { charge faisant intervenir plusieurs professionnels autour du patient. }\end{array}$ \\
\hline 6 & $\begin{array}{l}\text { Identifier les obstacles au travail } \\
\text { interdisciplinaire. }\end{array}$ & $\begin{array}{l}\text { Travaux de groupe pluriprofessionnel : « A partir de ces saynètes, de votre } \\
\text { expérience personnelle et de votre conception de la chose, quels sont, pour } \\
\text { vous, les obstacles au travail pluriprofessionnel ? » }\end{array}$ \\
\hline 7 & $\begin{array}{l}\text { Intervention d'un sociologue de la santé sur les conditions d'une bonne } \\
\text { coopération interprofessionnelle au service du patient. }\end{array}$ \\
\hline
\end{tabular}

authentiques. L'exemple du programme de la première session, centré sur la rencontre des autres professionnels permet d'illustrer cette construction pédagogique (tableau I). Des apports théoriques d'experts (sociologues, philosophes, etc.) dont le contenu était centré sur les interrogations des apprenants, des synthèses par les formateurs permettaient ensuite de décontextualiser. Des projets de groupe et des retours d'expérience étaient organisés par la suite au sein de chaque formation, même si la majeure partie de la recontextualisation devrait ensuite avoir lieu au cours de la vie professionnelle ${ }^{[14]}$.

Le travail de groupe en inter professionnalité a été la règle. Les apprenants devaient construire leurs apprentissages dans un environnement favorable au développement de compétences interprofessionnelles, analyser leurs propres conceptions et représentations. L'intégration de concepts nouveaux et une appréhension différente du monde étaient visées ${ }^{[13]}$.

\section{L'évaluation du dispositif de formation}

L'évaluation des apprentissages des apprenants ${ }^{[15]}$ était enchâssée dans les activités. Elle visait à analyser leurs processus cognitifs, afin de les accompagner dans leur construction de sens. Il s'agissait de restitutions de travaux, qui permettaient de rester dans des situations complexes et authentiques, de constructions de projets de groupes autour de situations cliniques complexes. Un retour écrit était demandé aux étudiants à l'issue de la formation, à remettre à leur formateur, pour documenter leurs apprentissages et une présentation de la formation aux autres étudiants de leur profession était prévue dans chaque institut de formation.

Concernant le dispositif de formation lui-même, une évaluation quantitative de la satisfaction des étudiants était réalisée à l'issue de chaque session, sur les 
différents aspects de la formation et sur les différentes activités pédagogiques organisées (par exemple le speed dating pédagogique ${ }^{[16]}$ ).

Une évaluation de la perception des étudiants concernant ce dispositif de formation et de leurs ressentis apparaissait indispensable pour compléter cette évaluation. La perception fait référence à la notion de réception et d'interprétation de stimuli sensoriels. Nous considérons la perception comme reliée au vécu des individus dans le sens des phénoménologues ${ }^{[17]}$.

Nous avons ainsi décidé de nous intéresser au discours des étudiants concernant ce dispositif de formation, deux ans après la fin de la formation. Le délai de deux années permettait d'analyser à distance ce que les étudiants pouvaient avoir retenu de l'expérience. L'objectif de notre étude était ainsi d'évaluer la perception des étudiants concernant ce séminaire et l'impact éventuel dans leur pratique professionnelle.

\section{Méthodes}

Une étude qualitative a été menée auprès d'étudiants ayant participé à cette formation. Il s'agissait d'explorer leurs perceptions de la formation interprofessionnelle. Deux chercheurs, médecins généralistes, ont ainsi réalisé des entretiens semi-directifs ; un des chercheurs était formateur lors du séminaire de formation.

Le choix d'une étude qualitative nous a paru indispensable pour explorer les perceptions des étudiants, en leur créant un espace de parole. Cette méthode est en effet utilisée pour l'observation d'un phénomène social en milieu naturel, traitant des données difficilement quantifiables ${ }^{[18]}$.

\section{Population de l'étude : échantillon et recrutement.}

L'étude a été réalisée auprès d'étudiants issus de la formation. L'objectif était d'avoir un corpus diversifié ${ }^{[19]}$ en rencontrant des étudiants de différentes professions.

Tous les étudiants ayant assisté au séminaire de formation entre octobre 2009 et octobre 2010 ont été contactés par courrier électronique. Nous demandions aux étudiants de nous répondre s'ils souhaitaient participer à une étude concernant le séminaire interprofessionnel. Les étudiants ayant répondu aux courriers électroniques ont été recontactés pour préciser les modalités de l'étude. Certains étudiants volontaires pour participer à l'étude ont été interrogés au cours d'entretiens semi-directifs réalisés par téléphone, du fait de leur dispersion géographique. Aucun étudiant en diététique n'a pu participer à l'étude. Plusieurs adresses électroniques de ces étudiants en diététique étaient erronées et les étudiants ayant répondu au courrier électronique initial ne pouvaient pas participer à l'étude, faute de disponibilité.

Le nombre d'entretiens n'était pas fixé au préalable. Ils ont été conduits jusqu'à saturation des données $^{[18]}$, c'est-à-dire lorsqu'aucun élément nouveau n'était identifiable dans les verbatims des entretiens. Seize étudiants ont été interrogés par téléphone entre octobre 2012 et août 2013 (tableau II). Ils représentaient donc sept professions sur les huit présentes initialement au cours de la formation.

\section{Collecte des données}

Le guide d'entretien a été élaboré après réalisation d'entretiens exploratoires (tableau III). Après avoir expliqué l'objet de l'étude aux participants, nous les interrogions sur la formation interprofessionnelle au sein de leur cursus. Nous souhaitions préciser les raisons de leur participation à la formation, avant de les interroger sur les objectifs d'une telle formation. Les éléments positifs et ceux à améliorer étaient abordés. Dans un second temps, nous nous concentrions sur leur vie professionnelle actuelle et l'impact de la formation sur leur pratique.

\section{Analyse des données.}

Les entretiens ont été enregistrés après accord des participants puis retranscrits intégralement. Les propos ont été anonymisés.

L'analyse a été effectuée de manière indépendante par deux chercheurs. L'analyse a été conduite en plusieurs étapes dans le cadre d'une analyse 
Tableau II. Population de l'étude.

\begin{tabular}{|c|c|c|c|c|}
\hline Entretien & Profession & Genre & $\begin{array}{l}\text { Niveau d'étude au début } \\
\text { de la formation interprofessionnelle }\end{array}$ & $\begin{array}{l}\text { Durée de l'entretien } \\
\text { (en minutes) }\end{array}$ \\
\hline E1 & Sage femme & $\mathrm{F}$ & $2^{\mathrm{e}}$ année & 41 \\
\hline E2 & Pharmacien & $\mathrm{F}$ & $5^{\mathrm{e}}$ année filière officine & 42 \\
\hline E3 & Masseur-kinésithérapeute & M & $2^{\mathrm{e}}$ année & 45 \\
\hline E4 & Masseur-kinésithérapeute & $\mathrm{F}$ & $2^{\mathrm{e}}$ année & 44 \\
\hline E5 & Masseur-kinésithérapeute & $\mathrm{F}$ & $2^{\mathrm{e}}$ année & 35 \\
\hline E6 & Pharmacien & $\mathrm{F}$ & $5^{\mathrm{e}}$ année filière officine & 40 \\
\hline E7 & Sage femme & $\mathrm{F}$ & $2^{\mathrm{e}}$ année & 35 \\
\hline E8 & Médecin généraliste & $\mathrm{F}$ & $\begin{array}{l}4^{\mathrm{e}} \text { semestre d'internat (soit } 2^{\mathrm{e}} \text { année d'internat de } \\
\text { médecine générale) }\end{array}$ & 44 \\
\hline E9 & Médecin généraliste & M & $\begin{array}{l}4^{\mathrm{e}} \text { semestre d'internat (soit } 2^{\mathrm{e}} \text { année d'internat de } \\
\text { médecine générale) }\end{array}$ & 43 \\
\hline E10 & Sage femme & $\mathrm{F}$ & $2^{\mathrm{e}}$ année & 37 \\
\hline E11 & Ergothérapeute & $\mathrm{F}$ & $2^{\mathrm{e}}$ année & 38 \\
\hline E12 & Podologue & M & $2^{\mathrm{e}}$ année & 41 \\
\hline E13 & Infirmier & $\mathrm{F}$ & $2^{\mathrm{e}}$ année & 30 \\
\hline E14 & Ergothérapeute & $\mathrm{F}$ & $2^{\mathrm{e}}$ année & 35 \\
\hline E15 & Infirmier & $\mathrm{F}$ & $2^{\mathrm{e}}$ année & 36 \\
\hline E16 & Infirmier & $\mathrm{F}$ & $2^{\mathrm{e}}$ année & 20 \\
\hline
\end{tabular}

Tableau III. Guide d'entretien.

Je suis ....... et j'aimerais évoquer avec vous l'expérience de formation interprofessionnelle à laquelle vous avez assisté entre octobre 2009 et octobre 2010.

Question «brise-glace» :

Pourriez-vous me raconter comment vous avez vécu cette formation?

I La formation dans le cursus

Quelle(s) étai(en)t la (les) raison(s) qui vous ont poussé à vous inscrire à cette formation ?

Quels étaient selon vous les objectifs d'une formation interprofessionnelle?

Quels étaient les points positifs et négatifs de la formation telle que vous l'avez vécue?

II La formation dans la vie professionnelle

Vous êtes maintenant diplômé. Quel est actuellement votre type exercice?

Quel a été selon vous l'impact de la formation dans votre cursus ? Sur votre exercice professionnel ?

Quelles seraient les perspectives des formations de ce type dans l'avenir? (place dans la formation initiale, et continue) 
Tableau IV. Les thèmes issus de l'étude qualitative.

\begin{tabular}{|l|}
\hline La formation interprofessionnelle dans les cursus de formation \\
Les objectifs spécifiques et le contenu pédagogique de la formation interprofessionnelle \\
Un impact sur l'exercice professionnel \\
- Une volonté de développer le travail collaboratif \\
- Des professionnels engagés dans des dynamiques interprofessionnelles. \\
- Une ouverture d'esprit, un changement de regard
\end{tabular}

thématique manuelle ${ }^{[18]}$. Les entretiens ont été lus intégralement à plusieurs reprises. Une première analyse de codage ouvert a été menée, sans aucune grille d'analyse établie au préalable. Ces éléments ont fait l'objet de discussions entre les chercheurs impliqués. Ensuite, selon un processus itératif de mise en commun et de discussion des analyses des deux chercheurs, un cadre d'analyse a été construit, permettant d'identifier des thèmes et des sousthèmes d'analyse. Ce cadre d'analyse a permis d'étudier l'ensemble des entretiens.

Les résultats ont également été présentés à deux étudiants ayant participé à l'étude afin qu'ils puissent donner leurs rétroactions.

\section{Résultats}

Plusieurs thèmes ont émergé de cette étude. Nous présentons ici les principaux résultats de cette étude (tableau IV).

La formation interprofessionnelle dans les cursus de formation

Les étudiants, quelle que soit leur origine professionnelle, insistaient sur le caractère novateur de la formation. Leur cursus habituel se déroulait sans contact avec les autres professionnels de santé. Une étudiante parlait de formations «tubulaires » qui, selon elle, renforcent l'incompréhension entre professionnels de santé. Le cloisonnement entre professions est vécu comme un frein pour développer des pratiques collaboratives.

«Dans notre formation nous n'avions pas eu l'occasion de rencontrer d'autres étudiants, à part les kinés et les podologues qui sont dans la même école que nous. Les autres métiers, on ne les connaissait pas tous. On parle de travailler ensemble pour les patients mais on n'avait pas eu de formation en commun. »E14, ergothérapeute

Selon les étudiants, cette absence de formation commune avait des conséquences sur leurs pratiques. Elle les empêchait de prendre en charge de façon globale les patients, en ne s'intéressant qu'à leurs seules compétences biocliniques. Le cloisonnement est renforcé par une mise en concurrence entre futurs professionnels.

«Oui, rencontrer les autres et puis voir vraiment comment on peut être amené à organiser ces choses là. Parce que c'est vrai que nous on n'est pas du tout formé. Sage-femme c'est un peu autocentré, on est très peu amené à aller vers les autres en ambulatoire. En hospitalier ça va, mais en ambulatoire...»E1, sage-femme

La méconnaissance des autres professions, l'absence de rencontre pouvait être un frein pour le suivi des patients. Ainsi, cette formation était souvent la première occasion de rencontrer d'autres futurs professionnels, de se former ensemble et d'appréhender la pluri professionnalité.

Les objectifs spécifiques et le contenu pédagogique de la formation interprofessionnelle

Selon les étudiants, l'objectif principal d'une telle formation était la découverte des autres et de leurs compétences.

"Et bien ce qu'ils ont réussi à faire sur six jours c'est à nous faire travailler ensemble, à collaborer. C'est vraiment ça qu'on pense voilà, à demander l'avis de la personne la mieux renseignée sur le sujet, 
qu'on travaille vraiment ensemble autour $d u$ patient. » E6, pharmacien

Ils insistaient sur la nécessité d'apprendre à se connaître afin de collaborer dans leur pratique future. La construction de la formation semblait avoir favorisé ces échanges et ces découvertes mutuelles. Ils découvraient que l'autre (professionnel) a une place essentielle dans la prise en charge du patient. La question du respect était centrale.

"Apprendre à se connaître pour se respecter, c'est vraiment ça. C'est vraiment le point que j'ai assimilé je crois. Il faut apprendre à se connaître aussi bien sur les compétences et savoir ce que l'autre fait que sur le plan relationnel. » E13, infirmier.

La première étape pour collaborer consistait selon eux à développer une relation de qualité avec l'autre professionnel. Au-delà du premier contact, les questions des représentations et de l'identité professionnelle émergeaient. La première session de la formation était consacrée à ces notions. Cette première approche marquait les esprits. Déconstruire les a priori apparaissait essentiel dans une logique de travail collaboratif.

"Je pense qu'un des objectifs c'est notamment de casser un peu certains préjugés [...] casser des idées reçues qu'on peut avoir sur chacune des filières, parce que justement on travaille ensemble mais un peu en parallèle finalement. Du coup il y a toujours une espèce de barrière les uns avec les autres. Et justement ce type de séminaire ça permet de casser ces barrières-là et de rentrer davantage en interaction les uns avec les autres. »E9, médecin généraliste

L'importance des représentations dans les relations qu' ils pouvaient développer avec les autres professionnels était soulignée. La notion de hiérarchie au sein des professions de santé était d'ailleurs réinterrogée.

"Mais on a des idées et des a priori sur les gens, et ça a permis de faire tomber tout ça, enfin surtout les professions médicales, je les voyais plus fermées que ça en fin de compte. Enfin, les professions paramédicales comme nous en fait, on n'avait pas de souci; je pensais bien qu'on allait bien s'entendre, mais c'était médecine, pharma, je pensais que ça allait être beaucoup plus difficile avec eux. »E12, podologue
Les étudiants insistaient sur l'image qu'ils avaient des professions de médecin ou de pharmacien où les rapports hiérarchiques étaient la norme. La rencontre avec de jeunes professionnels semblait propice à favoriser les échanges. Ils avaient découvert qu'il était possible de travailler avec les médecins dans une relation plus égalitaire. La notion de génération était néanmoins soulignée, avec l'idée que les jeunes professionnels étaient plus disposés au dialogue et au travail en inter professionnalité.

En fonction de leur future profession, les étudiants méconnaissaient souvent les compétences des autres professionnels. Les champs de compétences partagées étaient décrits comme d'importantes sources de tensions.

"Et ce problème de compétences partagées, dans nos formations en gros on nous l'apprend sur le mode concurrence, plutôt que de nous apprendre à travailler ensemble à partager ses compétences. » E7, ergothérapeute

Les temps informels (temps de pause, temps de repas) étaient essentiels pour mieux comprendre l'autre professionnel et ses compétences. Ils permettaient de considérer la place de chacun auprès du patient.

Cette place accordée au patient a été soulignée par plusieurs étudiants. Un temps de discussion avec des patients souffrant de pathologies chroniques, par groupes pluri professionnels, était organisé. C'était l'occasion de comprendre comment les patients vivaient avec leurs pathologies et ce qu'ils attendaient de la pluri professionnalité.

" J'avais trouvé ça super intéressant.[...] la rencontre [avec un patient] et puis les témoignages. Et puis c'est vrai qu'on se remet vraiment en question quand il nous dit à quel niveau il était satisfait ou pas et qu'il fallait plus l'écouter et prendre en compte que c'était une personne avec une vie, une vie de famille et une vie de couple. [...] C'était remettre le côté humain en priorité par rapport au métier. »E2, pharmacien

Ces rencontres semblaient avoir permis aux étudiants de changer leur regard sur les patients, sur leurs pathologies. Le patient devenait central dans une prise en charge holistique. 
Un impact sur l'exercice professionnel

Trois éléments concernant l'impact de cette formation ressortaient dans la manière dont était évoqué l'exercice professionnel.

\section{Une volonté de développer le travail collaboratif}

À l'issue du séminaire, les étudiants envisageaient différemment leur rôle auprès des patients.

"Le séminaire, oui, ça va changer ma façon de pratiquer. Finalement ça rend plus humble je trouve [rires]. On se dit qu'on n'est pas tout-puissant, qu'on a nous aussi droit à l'erreur, qu'on ne sait pas tout et finalement ça sert vraiment de se tourner vers des collègues pour avoir leur aide, c'est vraiment acquis, ça permet d'éviter au patient de stagner trop longtemps. Donc oui je pense que ça va plus m'inciter à aller vers les autres. » E5, masseur-kinésithérapeute.

De nombreux étudiants expliquaient vouloir favoriser le travail collaboratif. Ils cherchaient des structures hospitalières ou ambulatoires leur permettant de travailler avec d'autres professionnels de santé.

"C'est vrai que d'emblée j'ai essayé de mettre un peu en application ce côté, de montrer aux autres que j'ai envie de travailler avec eux [...] d'avoir un dialogue avec les autres professionnels de santé qui me demandent mon avis. Donc oui, je pense que j'ai gagné beaucoup en osant poser des questions aux autres professionnels sans me dire "c'est moi le médecin, je n'ai pas à poser les questions » en fait» E8, médecin généraliste

Lorsqu'ils abordaient leur mode d'exercice actuel, tous les interviewés exposaient leurs fonctions mais décrivaient également la composition de l'équipe avec laquelle ils travaillaient. Ils parlaient régulièrement des liens qu'ils entretenaient avec les autres professionnels et soulignaient leur volonté de comprendre leur fonction. Le mode d'exercice pluri professionnel est considéré par la majorité des étudiants comme un objectif dans leur carrière professionnelle. Ils ne considéraient plus leur simple rôle professionnel mais décrivaient leurs fonctions au sein d'une équipe, où chacun prend sa place.

\section{Des professionnels engagés dans des dynamiques interprofessionnelles}

Les étudiants interrogés sont nombreux à proposer des projets de travail en inter professionnalité, à initier une réflexion ou à prendre une part active dans les projets proposés. Des professionnels impliqués dans des dynamiques interprofessionnelles semblent émerger. Le séminaire interprofessionnel n'explique pas à lui seul cette attitude mais semble avoir contribué à faire germer des projets.

"Est né depuis 2 ans un projet de santé plus global sur la commune. Au début, on a été vers les pharmaciens en premier puis après au sein du cabinet on s'est posé la question de comment développer de nouveaux modes de rémunération, parce qu'on est tous un peu dans l'idée qu'il faut changer l'organisation du travail en libéral, le paiement à l'acte ça nous semble un peu désuet. [...] On a contacté l'ARS [Agence Régionale de Santé], on a pensé maison de santé pluridisciplinaire et puis finalement avec l'aide de l'ARS [...] on a contacté les autres professionnels, on a fait des réunions. Et au final de tout ça est née tout récemment une association de professionnels qui regroupe à peu près les $3 / 4$ des professionnels de la commune. " E8, médecin généraliste

Cette étudiante témoigne ainsi de l'émergence d'un projet avec d'autres professionnels de santé lors de son installation. La formation inter professionnelle, le choix de sa thématique de thèse sur les regroupements pluri professionnels ont influé sur ce projet. La formation rendait possible une projection concrète de travail collaboratif.

\section{Une ouverture d'esprit, un changement de regard}

De nombreux étudiants ont fait part de leur changement de regard dans leur conception de l'identité professionnelle.

"Ce que ça a changé...mais c'est surtout ma vision des autres je pense. Parce que du coupje connais mieux chaque profession et que je vais, enfin, c'est un peu comme le racisme quoi quand on connaît mieux les autres et bien on a moins peur d'aller voir. Et ça m'a aidé déjà à communiquer avec les professionnels qui m'entourent. »E4, masseur kinésithérapeute. 
Initialement, ils concevaient leur profession à partir de leurs propres compétences. La formation a modifié la vision de leur exercice. Ils n'envisageaient plus de prendre seul en charge un patient mais bien de s'intégrer à une équipe travaillant en inter professionnalité, que ces collaborations soient formalisées ou non. Par ailleurs, la rencontre avec les patients semblait modifier la représentation de leur propre rôle. Le patient devait, en fonction de ses besoins, de ses aspirations, choisir le mode de suivi, l'équipe de soignants travaillant dans une dynamique collaborative afin d'améliorer sa qualité de vie.

\section{Discussion}

Notre étude s'intéresse à la perception des étudiants en formation initiale de santé concernant une formation interprofessionnelle. Les limites de cette analyse sont à noter. Les étudiants contactés étaient réceptifs à notre démarche et volontaires pour participer à l'étude. En l'occurrence il s'agit d'un échantillon de disponibilité, ce qui constitue une limite théorique au regard du critère de crédibilité des données. Ainsi, l'absence d'entretiens auprès de diététiciens limite la diversité du corpus, qui reste cependant varié en termes de professions. Concernant l'objectivité de notre étude ${ }^{[20]}$, la fonction de formateur d'un des chercheurs au moment de la formation est à souligner et peut constituer un biais dans les réponses des étudiants, même si les thèmes identifiés étaient semblables dans les deux types d'entretiens. Le fait que les deux chercheurs soient médecins généralistes, eu égard aux dimensions hiérarchiques qui peuvent exister dans le travail des professionnels de santé, constitue aussi un biais même si cela n'a pas été précisé spécifiquement en début d'entretien. La fidélité de l'étude ${ }^{[20]}$ est néanmoins renforcée par l'implication de deux chercheurs. La validation des résultats ${ }^{[21]}$ par deux répondants est à souligner. Cette étude a été réalisée avec une triangulation des chercheurs mais seuls des entretiens individuels ont été menés. D'autres méthodes d'étude pourraient être utilisées pour renforcer la validité interne des résultats ${ }^{[18]}$. Néanmoins, l'implication des chercheurs dans le développement de la formation interprofessionnelle renforce cette validité ainsi que l'analyse des résultats qui concorde avec d'autres études menées à l'issue de formations interprofessionnelles ${ }^{[22,23,24]}$. Concernant la validité externe $^{[20]}$ des résultats, la saturation des données après réalisation de 16 entretiens est à noter. D' autres études pourraient être menées dans des contextes différents (autres villes, professions impliquées différentes) pour renforcer cet aspect.

Si des limites sont à noter concernant cette étude, son intérêt est renforcé par la réalisation d'entretiens deux ans après la formation. Les étudiants interrogés ont démarré leur exercice professionnel ce qui leur permet de réfléchir, à distance du séminaire, à leur pratique et aux apports de la formation. L'inter professionnalité est peu abordée au sein de leurs cursus. Cette formation apparaitt donc novatrice. C'est un lieu de rencontre entre futurs professionnels, qui permet de s'interroger sur les notions d'identité professionnelle et de représentations. Selon les étudiants, les objectifs de formations inter professionnelles sont nombreux, à la fois en termes de rencontre des autres professionnels, de découverte des compétences de chacun mais aussi de développement de relations plus égalitaires entre les différents acteurs de santé. Les méthodes pédagogiques utilisées semblent promouvoir de telles rencontres ${ }^{[16]}$. Si l'impact d'une telle formation est difficile à évaluer, les discours des étudiants mettent en exergue une nette volonté de développer le travail collaboratif. Les professionnels interrogés sont souvent engagés dans des dynamiques interprofessionnelles et le changement de regard sur la pratique est souligné. Pour eux, les formations interprofessionnelles sont essentielles pour développer des pratiques collaboratives et doivent être promues.

L'existence d'un tel dispositif de formation est une avancée. Le développement des compétences de collaboration professionnelle est nécessaire dès la formation initiale pour proposer des soins de qualité. Les étudiants rencontrés insistent sur l'intérêt de telles formations dans leur cursus. La stratégie nationale de santé, définie récemment en France ${ }^{[25]}$, préconise de s'appuyer sur des équipes de soins de proximité pluriprofessionnelles. Ceci implique de développer des formations interprofessionnelles, que 
ce soit en formation initiale ou continue. Ce type de formation est déjà très développé dans de nombreux pays, en particulier aux Etats-Unis, au Canada et au Royaume-Uni ${ }^{[26]}$.

Les objectifs de ce dispositif de formation, cités par les étudiants interrogés, font écho à ceux retrouvés dans la littérature ${ }^{[27]}$. Il s' agit notamment de travailler sur les représentations professionnelles qui peuvent freiner le développement de pratiques collaboratives ${ }^{[28]}$. Le renforcement de la communication inter professionnelle est poursuivi ainsi que la promotion du travail collaboratif au sein des équipes de soins ${ }^{[22]}$.

L'attention doit être portée au réalisme des situations proposées ${ }^{[23]}$ au sein de ces formations. L'authenticité des scénarios pédagogiques, voulue par les formateurs, était un atout. L'intégration de ces formations dans le curriculum est nécessaire ${ }^{[24]}$. Des activités d'enseignement et d'apprentissage en inter professionnalité pourraient être proposées tout au long du cursus.

Rencontrer des patients en dehors d'un cadre de soins et échanger autour de leur vision de la fonction de soignants et de l'inter professionnalité apparaît essentiel pour permettre un apprentissage basé sur l'acquisition de compétences. Plusieurs étudiants témoignent d'une modification de leur perception, passant d'un regard porté sur «une maladie » ou « un malade» à la découverte d'une personne. La volonté de rendre les patients acteurs de leur prise en charge en particulier ceux qui souffrent de pathologies chroniques impose ce changement de regard. Il s'agit d'orienter la pratique vers une logique communautaire de dispensation des soins ${ }^{[29]}$ et d'ancrer les apprentissages dans la réalité des patients.

De nombreux étudiants apparaissent engagés dans des dynamiques de travail inter professionnel, que ce soit dans le cadre d'exercice ambulatoire (projets de maisons de santé par exemple) ou dans un cadre hospitalier. Plusieurs étudiants de différentes professions expriment ainsi une nette volonté de changement, une motivation et un engagement pour mettre en place des dispositifs de soins innovants. Cela nous interroge sur le lien entre la formation et cette émergence de ce que nous pouvons qualifier de leadership, au sens de capacité de l'individu à mobiliser un groupe en faveur d'un objectif prédéfini et bien identifié ${ }^{[30]}$. Le leadership dans le milieu de la santé est vu comme un levier d'action pour envisager des changements dans l'organisation et la dispensation des soins ${ }^{[30]}$. Dans le développement de l'inter professionnalité c'est un facteur important de changement. Mais des entraves existent, en particulier les relations hiérarchiques qui persistent dans le monde de la santé, particulièrement entre médecins et autres professions de santé ${ }^{[31]}$. Faire prendre conscience des représentations que les étudiants ont des autres professions, lors de cette formation interprofessionnelle, est une première étape. Travailler sur les modes de relation qui existent entre professionnels de santé, en intégrant les notions de pouvoir et de conflit pour développer des relations plus égalitaires pourrait constituer une perspective intéressante dans le cadre de cette formation.

\section{Conclusion}

Apprendre à travailler ensemble est indispensable pour les futurs professionnels de santé. L'intérêt de formations centrées sur la problématique de l'inter professionnalité, permettant de rencontrer les autres professionnels de santé, déconstruire les représentations et travailler dans une logique de collaboration inter professionnelle est souligné par les étudiants. D'autres études devront être poursuivies concernant l'acquisition de compétences à la collaboration interprofessionnelle, le développement de relations plus égalitaires entre professionnels et l'impact sur la qualité des soins, pour promouvoir ce type de formation et les intégrer au curriculum des étudiants en santé.

\section{Valorisation scientifique}

Ce travail a fait l'objet d'un mémoire dans le cadre d'un Diplôme inter-universitaire de pédagogie et de communication médicale (Universités d'Angers, Bretagne occidentale, Nantes et Rennes)

\section{Remerciements}

A l'ensemble de l'équipe des formateurs de Rennes : les formateurs de l'école de diététique, de l'Institut de 
Formation en Podologie Ergothérapie et Kinésithérapie (IFPEK), des Instituts de formation en soins infirmiers (IFSI) du Centre Hospitalier Guillaume Régnier de Rennes et de Saint Malo, de l'Ecole de sage-femme du Centre hospitalier universitaire de Rennes et de la Faculté de pharmacie de l'Université de Rennes 1, sans lesquels cette formation n'aurait pas eu lieu

\section{Contributions}

Laure Fiquet a participé à la conception du protocole de recherche, à la réalisation de l'étude, à l'interprétation des données et à la rédaction du manuscrit. Sandrine Huge a participé à la conception du protocole de recherche, à la réalisation de l'étude, à l'interprétation des données et à la relecture du manuscrit. Françoise Annezo a eu l'initiative de la proposition du dispositif de formation et a été responsable de sa conception ; elle a participé à l'écriture et à la relecture du manuscrit. Anthony Chapron et Emmanuel Allory ont participé à la conception du protocole de recherche et à la relecture du manuscrit. Pierric Renaut a participé à la conception du dispositif de formation et du protocole de recherche, et à la relecture du manuscrit.

\section{Déclaration d'intérêt}

Aucun auteur ne déclare de conflit d'intérêt en lien avec le contenu de cet article.

\section{Approbation éthique}

Non sollicitée

\section{Références}

1. Bourgueil Y, Le Fur P, Mousquès J, Yilmaz E. La coopération entre médecins généralistes/infirmières améliore le suivi des patients diabétiques de type2. IRDES Questions de santé 2008;136:1-8.

2. Elbaum M (sous la direction de). Enjeux économiques des coopérations entre professionnels de santé. Paris : Haute autorité de santé. 2007 [On-line] Disponible sur: http://www.has-sante.fr/portail/ upload/docs/application/pdf/pratiques_actuelles_ de_cooperation.pdf

3. D'amour D, Sicotte C, Lévy R. L'action collective au sein d'équipes interprofessionnelles dans les services de santé. Sciences sociales et santé 1999;17: 67-94.

4. San Martin Rodriguez L, Beaulieu MD, D'Amour D, Ferrada-Videla M. The determinants of successful collaboration: A review of theoretical and empirical studies. J Interprof Care 2005;19 (suppl 1):132-47

5. Contribution collective de la conférence pour le consensus mondial sur la responsabilité sociale des facultés de médecine. Consensus mondial sur la responsabilité sociale des facultés de médecine. Pédagogie Médicale 2011;12:37-48

6. Collectif : Mission nationale, Evaluation des compétences professionnelles des métiers de la santé ; Collège National des Généralistes Enseignants (CNGE) ; Conseil National de l'Ordre des SagesFemmes (CNOSF) ; Collectif des Associations et Syndicats de Sages-Femmes (CASSF); Collège National des Gynécologues et Obstétriciens Français (CNGOF). Référentiel métier et compétences. Médecins généralistes, sages-femmes et gynécologues obstétriciens. Paris : Berger-Levrault, 2010.

7. CAIPE. Centre For The Advancement Of Interprofessional Education. Definition of Interprofessional education. 2002. [On-line] Disponible sur : http:// www.caipe.org.uk/about-us/defining-ipe/.

8. Harden RM. AMEE guide No. 12: Multiprofessional education: Part 1 effective multiprofessional education: a three-dimensional perspective. Med Teach 1998;20:402-8.

9. Organisation mondiale de la santé. Framework for Action on Interprofessional Education and Collaborative Practice. Genève: OMS, 2010.

10. Morin E. Sur l'interdisciplinarité. Bulletin Interactif du Centre International de Recherches et Études transdisciplinaires. 1994 [On-line]. Disponible sur : http://ciret-transdisciplinarity.org/bulletin/ b2c2.php 
11. Dubar C, Tripier P. Introduction. In : Dubar C, Tripier P. Sociologie des professions. ( $2^{\mathrm{e}}$ édition). Paris : Armand Colin, 2005:1-15.

12. Jouquan J, Bail P. A quoi s'engage-t-on en basculant du paradigme d'enseignement au paradigme d'apprentissage? Pédagogie Médicale 2003;4:163-75.

13. Bernard JL, Reyes P. Apprendre en médecine (2e partie). Pédagogie Médicale 2001;2:235-41.

14. Vanpee D, Frenay M, Godin V, Bédard D. Ce que la perspective de l'apprentissage et de l'enseignement contextualisés authentiques peut apporter pour augmenter la qualité pédagogique des stages d'externat. Pédagogie Médicale 2010;10:253-66.

15. Jouquan J. L'évaluation des apprentissages des étudiants en formation médicale initiale. Pédagogie Médicale 2002;3:38-52.

16. Renaut P, Fiquet L, Allory E, Chapron A, Hugé S, Annezo F. Le speed dating : une innovation pédagogique pour enseigner la collaboration interprofessionnelle. Exercer 2015;118:184-90.

17. Raynal F, Rieunier A. Pédagogie, dictionnaire des concepts clés. Apprentissage, formation, psychologie cognitive. $7^{\mathrm{e}}$ édition. Paris : ESF éditeurs. 2009.

18. Blanchet A, Gotman A. L'enquête et ses méthodes. L'entretien. $2^{\mathrm{e}}$ édition. Paris : Armand Colin. 2009.

19. Savoie-Zajc L. L'échantillonnage en recherche qualitative. Recherches qualitatives. Hors-série. Actes du colloque recherches qualitatives : les questions de l'heure 2007;5:99-111.

20. Drapeau M. Les critères de scientificité en recherche qualitative. Pratiques psychologiques 2004;10: 79-86.

21. Muchielli A. Dictionnaire des méthodes qualitatives en sciences humaines. $3^{\mathrm{e}}$ édition Paris : Armand Colin. 2011.

22. Rosenfield D, Oandasan I, Reeves S. Perceptions versus reality: a qualitative study of students'expectations and experiences of interprofessional education. Med Educ 2011;45:471-7.
23. Ruebling I, Pole D, Breitbach AP, et al. A comparison of student attitudes and perceptions before and after an introductory interprofessional education experience. J Interprof Care 2014;28:23-7.

24. Reeves S, Goldman J, Freeth D, Zwarenstein M. Interprofessional education: effects on professional practice and health care outcomes. Cochrane Database Syst Rev 2013:1-47.

25. Cordier A (sous la direction de) Comité des sages. Un projet global pour la stratégie nationale de santé. 19 propositions du comité des "sages". La Documentation française. 2013 [On-line] Disponible sur : http://www.ladocumentationfrancaise.fr/var/storage/rapports-publics/134000632.pdf

26. Rodger S, Hoffman SJ. Where in the world is interprofessional education? A global environmental scan. J Interprof Care 2010;24:479-91.

27. Organisation mondiale de la santé. Framework for Action on Interprofessional Education and Collaborative Practice. OMS: Genève, 2010.

28. Sarradon-Eck A, Vega A, Faure M, Humbert-Gaudart A, Lustman M, Créer des liens : les relations soignants-soignants dans les réseaux de soins informels. Revue d'Épidémiologie et de santé publique 2008;56:197-206.

29. Pestiaux D, Boelen C, Nawar T, Ladner J. Responsabilité sociale des facultés de médecine. In: Parent F, Jouquan J. Penser la formation des professionnels de la santé. Une perspective intégrative. Bruxelles : De Boeck. 2013:89-112.

30. Arweiler D, Noyeau E, Charlin B, Millette B, Hodges B. Le leadership comme facteur de changement dans le champ de la santé: rôle de l'éducation médicale. Pédagogie Médicale 2010;11:239-53.

31. Olson RE. How would an egalitarian health care system operate? Power and conflict in interprofessional education. Med Educ 2015;49:353-4.

Correspondance et offprints: Laure Fiquet, Département de médecine générale, Université de Rennes 1, 2 Av. du Pr Léon Bernard, 35043 Rennes Cedex, France.

Mailto : laure.fiquet@univ-rennes1.fr 